\title{
ĐÁNH GIÁ HIỆU QUẢ CHƯƠNG TRÌNH CAN THIỆP HƯớNG DẪN SỬ DUUNG THUỐC CHO NGƯờI BÊ̂NH TĂNG HUYẾT ÁP TẠI BỆNH VIỆN ĐA KHOA THỐNG NHẤT TỈNH ĐỒNG NAI NĂM 2020
}

\section{TÓM TẮT}

Mục tiêu: Đánh giá hiệu quả của Chương trình can thiệp hướng dẫn sử dụng thuốc (CTHDSDT) cho NBTHA tại Bệnh viện đa khoa Thống Nhất tỉnh Đồng Nai năm 2020. Phương pháp nghiên cứu: Nghiên cứu tiến hành áp dụng và đánh giá hiệu quả của Chương trình CTHDSDT, thông qua so sánh mức độ tuân thủ sử dụng thuốc của NBTHA trước và sau can thiệp theo thang điểm MMAS-8. Kết quả: Mẫu nghiên cứu có 128 NBTHA tham gia Chương trìnhCTHDSDT, thời gian trung bình tư vấn trực tiếp tại bệnh viện cho 1 NBTHA trước và sau Chương trình CTHDSDT là 12,3 $\pm 4,5$ phút và $6,9 \pm 2,0$ phút $(p<0,05)$; tỷ lệ NBTHA tuân thủ sử dụng thuốc tăng $20,3 \%(p<0,05)$. Kết luận: Nghiên cứu cho thấy hiệu quả củaChương trình CTHDSDTcho NBTHA trong việc cải thiện tỷ lệ tuân thủ sử dụng thuốc của người bệnh và thời gian tư vấn sử dụng thuốc, có thể triển khai áp dụng cho các đối tượng khác tại bệnh viện.

Tư khóa: Tư vấn, người bệnh tăng huyết áp, sự tuân thủ điều trị, can thiệp.

\section{SUMMARY}

ASSESSMENT OF THE DRUGS COUNSELING INTERVENTION PROGRAM FOR HYPERTENSIVE PATIENTS AT THONG NHAT GENERAL HOSPITAL, DONG NAI PROVINCE IN 2020

Objectives: To assess the drugs counseling intervention program (DCIP) for hypertensive patients at Thong Nhat General Hospital, Dong Nai Province in 2020. Methods: A combined experimental and descriptive study was carried out to compare the amount of direct counseling time and the drug adherence of hypertensive patients before and after the DCIP by the MMAS- 8 scale. Results: A total of 128 hypertensive patients participated in the DCIP. The average counseling session time was $12.3 \pm 4.5$ minutes before the DCIP and $6.9 \pm 2.0$ minutes after the DCIP $(p<0.05)$. The number of patients with high adherence increased by $20.3 \%$ after the DCIP $(p<0.05)$. Conclusion: The drugs counseling intervention program at Thong Nhat General Hospital,

${ }^{1}$ Bệnh viện Thống Nhất Đồng Nai,

${ }^{2}$ Tập đoàn Y khoa Hoàn Mỹ

3 Đai hoc Y Dướ TP. Hồ Chí Minh

Chịu trách nhiệm chính: Hoàng Thy Nhạc Vũ

Email: hoangthynhacvu@ump.edu.vn

Ngày nhận bài: 4/3/2021

Ngày phản biên khoa hoc: 2/4/2021

Ngày duyệt bài: 3/5/2021
Bùi Mai Nguyệt Ánh ${ }^{1}$, Nguyễn Tấn Phúc ${ }^{1}$, Đào Duy Kim Ngà ${ }^{2}$, Hoàng Thy Nhạc Vũ $\tilde{u}^{3}$

Dong Nai province improved the drug adherence among hypertensive patients. In the future, the program may apply to other patients at the hospital.

Key words: Drugs education and counseling, hypertensive patient, drug adherence, intervention.

\section{I. ĐẶT VẤN ĐỀ}

Để đảm bảo an toàn và hiệu quả trong điêu tri bằng thuốc, người bênh (NB) cân nhân được sự tư vấn hướng dẫn sử dụng thuốc của cán bộ y tế một cách đây đủ, tạo cơ sở giúp họ thực hiện nhiệm vụ tuân thủ điều trị một cách tốt nhất. Bệnh viện đa khoa Thống Nhất tỉnh Đồng Nai (BVĐKTNĐN) có quy mô 1.000 giường bệnh, với khoảng 2.000 lượt khám ngoại trú mổi ngày, trong đó người bệnh tim mạch đến khám và điều tri chiếm số lướng nhiều nhất trong các nhóm bệnh. Trong năm 2019, số lượng người bệnh tăng huyết áp (NBTHA) chiếm $51 \%$ tổng số người bệnh điêu trị ngoại trú tại bệnh viện. NBTHA thường có nhiều bệnh đi kèm, được chỉ định sử dụng nhiêu thuốc trong thời gian dài. Việc tuân thủ điêu trị của người bệnh là một yếu tố then chốt để đạt được hiệu quả điều trị. Tuy nhiên, nhiều nghiển cứu cho thấy có nhiều yếu tố tác động đến sự tuân thủ điều trị của người bệnh $\operatorname{NBTHA}^{(3,5)}$. Hoạt động tư vấn sử dụng thuốc cho NB điêu trị ngoại trú là một hoạt động thường quy, được thực hiển tại BVĐKTNĐNnhằm hỗ trơ NB tuân thủ sử dụng thuốc. Tuy nhiên, việc thực hiện hoạt động tư vấn tại BVĐKTNĐN còn thiếu sự đồng bộ giữa các khoa phòng, chưa đat được hiêuu quả mong muốn do chưa có các quy trình chuẩn hóa và đồng nhất. Dưa vào nhu câu thực tế của NB, vào nguôn lực hiện có của BV, Ban giám đốc BVĐKTNĐNđã đồng ý xây dựng và triển khai Chương trình can thiệp hướng dẩn sử dụng thuốc cho NBTHA (Chương trình CTHDSDT) nhằm rút ngắn thời gian tư vấn tại bệnh viện, giảm thời gian chờ đợi của người bệnh; tăng tỷ lệ tuân thủ của NB. Nghiên cứu được thực hiện nhằm đánh giá hiệu quả bước đâu của Chương trình CTHDSDT, tạo cơ sở cho các hoạt động cải tiến chất lượng chuyên môn và chuẩn hóa hoạt động tư vấn sử dụng thuốc cho NB tại tại BVĐKTNĐN. 
II. ĐỐI TƯƠNGG VÀ PHƯƠNG PHÁP NGHIÊN CỨU

2.1. Mẫu nghiên cứu: Nghiên cứu chọn toàn bộ những NBTHA thuộc Hội khám Tim mạch tại BV ĐKTN ĐN năm 2020 để chọn vào tham gia nghiên cứu. Có 128 NBTHA đạt tiêu chí: từ 18 tuổi trở lên, đủ sức khỏe để trả lời khảo sát, có khả năng tham gia toàn bô các hoat động trong Chương trình CTHDSDT, và đồng ý tham gia vào nghiên cứu.

Hoat động của Chương trình CTHDSDT cho NBTHA tai BV ĐKTN ĐN:

- Tư vấn cho NBTHAtrực tiếp tại BV theo nội dung đã được soạn thảo, chuẩn hóa, và ban hành trong Sổ tay hướng dẫn (các nội dung lưu ý đối với người bệnh, tương tác thuốc, thời gian sử dụng, tác dụng phụ được khuyến cáo theo Dược thư quốc gia, thông tin nhà sản xuất, Micromedex, Uptodate).

- Tư vấn cho NB từ xa thống qua ứng dụng trên điện thoại: NB được hướng dẫn sử dụng ứng dụng "App Hội dược sĩ- thông tin Y dược" để tiếp tục nhận được tư vấn trong quá trình sử dụng thuốc tại nhà. Ứng dụng có các tính năng lựa chọn Dược sĩ tư vấn; Lưu trữ các thông tin về đơn thuốc, tiền sử bệnh; nhắc nhở thời gian uống thuốc; Yêu cầu tư vấn online về thuốc hoặc các vấn đề về sức khỏe thông qua ứng dụng).

2.2. Thiết kế nghiên cứu: Toàn bộ NBTHA sẽ được mời tham gia khảo sát hai lần trong thời gian từ tháng $8 / 2020$ đến tháng $11 / 2020$, lần 1 để thu thập thông tin cá nhân (giới tính, tuổi, trình độ học vấn, nơi sống, nghề nghiệp, số năm được chẩn đoán THA, các bệnh kèm theo, số thuốc được chỉ định) và thông tin liên quan đến tuân thủ điều trị; lần 2 chỉ thu thâp thông tin liên quan đến tuân thủ điều trị. Sau khi người bệnh tham gia khảo sát lần 1, 1 tháng sau sẽ được tư vấn sử dụng thuốc theo Chương trình CTHDSDT,
3 tháng tiếp theo được khảo sát lần 2. Hiệu quả Chương trình CTHDSDTđược đánh giá thông qua sự khác biệt về thời gian tư vấn trực tiếp và tỷ lệ tuân thủ sử dụng thuốc của NB trước và sau khi tham gia Chương trình CTHDSDT.

Sự tuân thủ điều trị thuốc được đánh giá theo thang đo MMAS- 8 (Medication adherence questionaire Morisky 8 - MMAS-8). MMAS-8 là một thang đo thông dụng được áp trong rất nhiều nghiên cứu về sự tuân thủ điều trị của NB mạn tính, đặc biệt là THA. Nghiên cứu chọn ngưỡng 6 của thang đo MMAS-8 để chia NB thành 2 mức đô là "có tuân thủ" ( $\geq 6$ điểm) và "không tuân thư" (<6 điểm) ${ }^{(6,8)}$, với ước tính độ nhạy trong việc xác định mức tuân thủ là $93 \%$, độ đặc hiệu là $53 \%$.

2.3. Xử lý thống kê: Phần mềm Excel và Stata được sử dụng để tổng hợp và phân tích dữ liệu. Thông tin được mô tả thổng qua tần số, tỷ lệ, giá trị trung bình( \pm độ lệch chuẩn), so sánh 2 tỳ lệ bằng phép kiểm Chi bình phương, so sánh 2số trung bình bằng phép kiểm t-test.

\section{KẾT QUẢ NGHIÊN CỨU}

3.1. Đặc điểm của mẫu nghiên cứu. Trong 128 NBTHA đạt các tiêu chí và được đưa vào nghiên cứu, $57,0 \%$ là nữ, độ tuổi trung bình là $60 \pm 6,7$ tuổi; $65,6 \%$ là lao động chân tay chiếm, $74,2 \% \mathrm{NB}$ ở thành thị. $71,1 \% \mathrm{NB}$ có thời gian mắc bệnh THA từ 10 năm trở lên, có $49 \%$ NB mắc từ 3 bệnh kèm trở lên. NB được chỉ định trung bình $5,4 \pm 1,5$ cho một đơn, trong đó có $78,1 \%$ NB được chỉ định trên 5 thuốc.

So sánh điểm MMAS-8 về sự tuân thủ sử dụng thuốc ở các nhóm NB khác nhau, kết quả ghi nhận ở hầu hết các nhóm điểm MMAS-8trung bình trước khi áp dụng Chương trình đều cao hơn sau khi áp dụng Chương trình CTHDSDT (Bảng 1).

Bảng 1. Đặc điểm của 128 người bệnh tăng huyết áp tham gia nghiên cứu

\begin{tabular}{|c|c|c|c|c|}
\hline Đặc điểm & $\begin{array}{l}\text { Số lượng } \\
(n=128)\end{array}$ & $(\%)$ & $\begin{array}{l}\text { Điếm MMAS-8 } \\
\text { khảo sát lân } 1\end{array}$ & $\begin{array}{c}\text { Điếm MMAS-8 } \\
\text { khảo sát lân } 2\end{array}$ \\
\hline Giới tính: & 55 & $(43,0)$ & $6,3 \pm 1,4$ & $7 \pm 1,2$ \\
\hline Nữ & 73 & $(57,0)$ & $6,3 \pm 1,4$ & $7 \pm 1,1$ \\
\hline Nhóm tuối: $\geq 60$ tuối & 76 & $(59,4)$ & $6,2 \pm 1,5$ & $6,4 \pm 1,3$ \\
\hline$<60$ tuối & 52 & $(40,6)$ & $6,4 \pm 1,3$ & $7,1 \pm 1,1$ \\
\hline Đăc điếm nghề nghiêp: Lao đông trí óc & 44 & $(34,4)$ & $6,0 \pm 1,4$ & $6,9 \pm 1,0$ \\
\hline Lao động chân tay & 84 & $(65,6)$ & $6,4 \pm 1,4$ & $6,7 \pm 1,4$ \\
\hline Thành thị & 95 & $(74,2)$ & $6,0 \pm 1,4$ & $6,8 \pm 1,2$ \\
\hline Nông thồn & 33 & $(25,8)$ & $7,2 \pm 1,0$ & $7,7 \pm 0,8$ \\
\hline Thời gian măc bệnh THA: <10 năm & 37 & $(28,9)$ & $5,9 \pm 1,3$ & $6,6 \pm 1,2$ \\
\hline$\geq 10$ năm & 91 & $(71,1)$ & $6,5 \pm 1,4$ & $7,2 \pm 1,1$ \\
\hline Số bênh kèm theo: 0-1 bênh & 22 & $(17,2)$ & $5,8 \pm 1,5$ & $6,7 \pm 1,2$ \\
\hline 2 bệnh & 43 & $(33,6)$ & $6,5 \pm 1,3$ & $7,1 \pm 1,1$ \\
\hline
\end{tabular}




\begin{tabular}{|c|c|c|c|c|}
\hline$\geq 3$ bệnh & 63 & $(49,2)$ & $6,3 \pm 1,5$ & $7,0 \pm 1,1$ \\
\hline Số thuốc trong đơn: < 5 thuốc & 28 & $(21,9)$ & $6,4 \pm 1,5$ & $7,0 \pm 1,2$ \\
\hline$\geq 5$ thuốc & 100 & $(78,1)$ & $6,2 \pm 1,4$ & $7,0 \pm 1,1$ \\
\hline
\end{tabular}

3.2. So sánh nội dung tư vấn, hướng dẫn cho NBTHA trước và sau khi triển khai Chương trình CTHDSDT

Nội dung tư vấn về "Chỉ định, liều dùng và thời gian sử dụng thuốc" đều được thực hiện đầy đủ cho $128 \mathrm{NB}$ trước và trong khi can thiệp. Việc triển khai Chương trìnhCTHDSDT cho thấy sự gia tăng số lượng NB được tư vấn về các nội liên quan đến "Tác dụng phụ của thuốc và cách xử trí"(48 vs 20), "Cách xử trí nếu quên thuốc" (99 vs 10),"Cách bảo quản thuốc" (13 vs 10), "Chế độ dinh dưỡng trong thời gian sử dụng thuốc" (8 vs 2).(Hình 1)

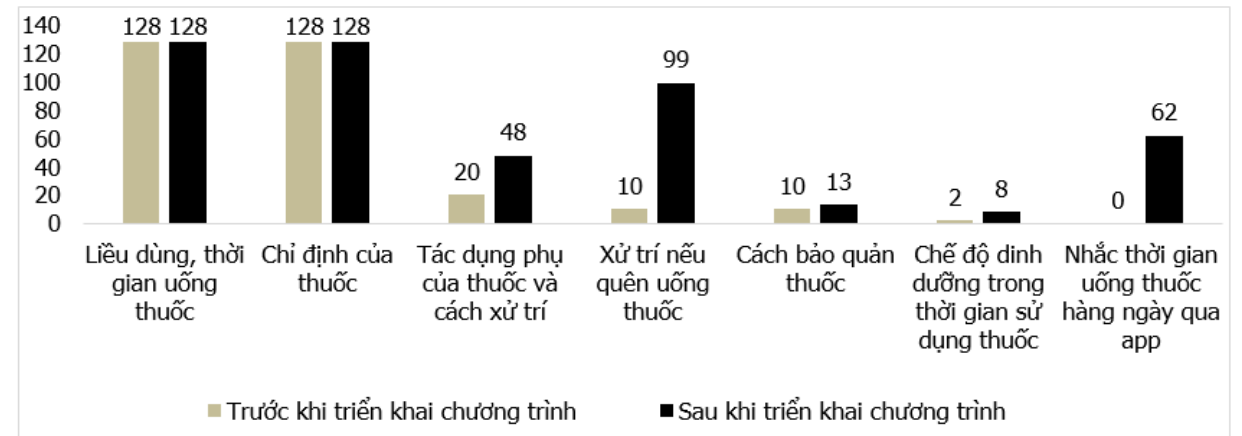

Hình 1. So sánh nội dung người bệnh tăng huyết áp được tư vấn, hướng dẫn sư dụng thuốc thời điểm trước và sau khi triển khai Chương trinh can thiệp hướng dẫn sử dụng thuốc

3.3. Hiệu quả Chương trình can thiệp hướng dẫn sử dụng thuốc cho NBTHA: Sau 3 tháng triển khai Chương trình CTHDSDTcho NBTHA, kết quả ghi nhậnthời gian trung bình tư vấn trực tiếp cho $1 \mathrm{NB}$ tại bệnh viện giảm $(12,3$ $\pm 4,5$ vs $6,9 \pm 2,0)$, tỷ lệ NB tuân thủ sử dụng thuốc tăng từ $64,1 \%$ lên $84,4 \%(p<0,05)$, điểm MMAS-8 trung bình của mẫu nghiên cứu tăng từ $6,29 \pm 1,4$ lên $7,0 \pm 1,0$ ( $p<0,05)$ (Bảng 2).

Bảng 2. Hiêuu quá Chương trình can thiệp hướng dẫn sử dụng thuốc cho người bệnh tăng huyêt áp

\begin{tabular}{|c|c|c|c|}
\hline $\begin{array}{c}\text { Tiêu chí đánh } \\
\text { giá }\end{array}$ & $\begin{array}{c}\text { Trước khi } \\
\text { triển } \\
\text { khai } \\
\text { CTHDSDT }\end{array}$ & $\begin{array}{c}\text { Sau khi } \\
\text { triển khai } \\
\text { CTHDSDT }\end{array}$ & p \\
\hline $\begin{array}{c}\text { Thời gian TB tư } \\
\text { vấn trực tiếpcho } \\
1 \text { NB taai bệnh } \\
\text { viện (phút) }\end{array}$ & $12,3 \pm 4,5$ & $6,9 \pm 2$ & 0,001 \\
\hline $\begin{array}{c}\text { Tý lệ NB tuân } \\
\text { thủ sử dụng } \\
\text { thuốc }\end{array}$ & & & \\
\hline Có tuân thư & $82(64,1 \%)$ & $108(84,4 \%)$ & $<0,001$ \\
\hline Không tuân thủ & $46(35,9 \%)$ & $20(15,6 \%)$ & \\
\hline $\begin{array}{c}\text { Điểm MMAS-8 } \\
\text { (Trung bình } \pm \text { SD) }\end{array}$ & $6,3 \pm 1,4$ & $7,0 \pm 1,0$ & $<0,001$ \\
\hline
\end{tabular}

IV. BÀN LUẬN

Nghiên cứu đã đánh giá được hiệu quả bước đầu của Chương trình CTHDSDTtại BV ĐKTNĐN năm 2020. Mẫu nghiên cứucó tỷ lệ nữ cao hơn nam, tỷ lệ người bệnh cao tuổi chiếm 59,4\%. Đặc điểm này tương đồng với đặc điểm của NBTHA trong nghiên cứu khác tại Việt Nam ${ }^{(1)}$. Do đặc thù CTHDSDTcó sử dụng công cụ hỗ trợ là điện thoại thông minh để tư vấn sử dụng thuốc từ xa, những NB quá lớn tuổi không thể tham gia vào CTHDSDT nên độ tuổi trung bình trong nhóm tham gia nghiên cứu nhỏ hơnđộ tuổi trung bình của NBTHA ở một nghiên cứu khác (NB cao tuổi chiếm $70 \%)^{(2)}$. Trước đây, hoạt động tư vấn và hướng dẫn sử dụng thuốc tại BV ĐKTNĐN đã được thực hiện nhưng chưa có quy trình cụ thể nên nội dung tư vấn còn chua dồng nhất. Sau khi xây dựng Chương trình CTHDSDT với những nội dung hướng dẫn, công cụ hỗ trợ và quy trình cụ thể, chất lượng của hoạt động tư vấn sửa dụng thuốc có sự thay đổi rõ̉ nét. Các nội dung được dược sĩ tư vấn nhiều hơn so với trước là "nhẩn biết và xử trí tác dung phu của thuốc", "xử trí nếu quên uống thuốc", "chế độ dinh dưỡng trong thời gian sử dụng thuốc", đặc biệt là khi sử dụng thuốc chống đồng.

Kết quả nghiên cứu ghi nhận thời gian tư vấn trung bìnhtrực tiếp cho 1 NB tại bệnh viện giảm đáng kể so với trước khi triển khai Chương trìnhCTHDSDT $(6,9 \pm 2$ phút vs $12,3 \pm 4,5$ phút, $\mathrm{p}<0,05)$. Thời gian tư vấn tại bệnh viện giảm do 
dược sĩ có thể tư vấn bằng điện thoại qua ứng dụng hỗ trợ nên NB không cần phải chờ đợi lâu ở bệnh viện để được tư vấn mà có thể hỏi các thông tin về thuốc khi ở nhà. Nhiềunghiên cứu trên thế giới đã chứng minh sự can thiêp của nhân viên y tế tác động tích cực lên sự tuân thủ sử dụng thuốc của NB. Nghiên cứu ở Canada năm 2014 về tác động của dược sĩ trong việc quản lý NBTHA, nhóm can thiệp có tỷ lệ tuân thủ tăng cao hơn so với nhóm chứng (15\% vs $2,2 \%)$ (7). Một nghiên cứu ở Nepal phỏng vấn 332 NBTHA cho thấy nhóm có sự tư vấn của dược sĩ có tỷ lệ tuân thủ cao hơn nhóm chứng $(86,15 \%$ vs $13,86 \%$ ) (8). Chương trình CTHDSDT cũng ghi nhận tác động tích cực của Dược sĩ trong việc giúp tăng tỷ lệ NB tuân thủ sử dụng thuốc.

\section{KẾT LUẬN}

Chương trình can thiệp hướng dẫn sử dụng thuốc cho NBTHA tại Bệnh viện đa khoa Thống Nhất tỉnh Đồng Nai bước đầu đạt được một số hiệu quả nhất định, tạo tiền đề cho việc tiếp tục triển khai và áp dụng chương trình này tại Bệnh viện.

\section{TÀI LIÊU THAM KHẢO}

1. Thái Khoa Bảo Châu (2016). Nghiên cứu tình hình sử dụng thuốc trong điều trị tăng huyết áp tại Bệnh viện trường đại học Y Dược Huế. Tạp chí Y Dược Huế số 32, trang 76-84.

2. Nguyễn Thị Thủy (2018). Khảo sát kiến thức về bênhh tăng huyết áp của bệnh nhân tăng huyết áp điêuu tri nội trú tai bênh viện quân y 103 năm 2017. Tạp chí Y Dược học quân sự, số 1, năm 2018, trang 29-35.

3. Ashp (1997). Medication Therapy and Patient Care: Organization and Delivery of ServicesGuidelines ASHP Guidelines on PharmacistConducted Patient Education and Counseling. American Journal of Health-System Pharmacy, vol. 54, No. 4. pp. 340-342.

4. Emilio Márquez-Contreras (2006). Efficacy of a Home Blood Pressure Monitoring Programme on Therapeutic Compliance in Hypertension: The EAPACUM-HTA Study. Journal of hypertension, Vol 24, pp 169-175.

5. Jean-Pierre Fina Lubaki (2009). Reasons for noncompliance among patients with hypertension at Vanga Hospital, Bandundu Province, Democratic Republic of Congo: A qualitative study. African Journal of Primary Health Care \& Family Medicine, Vol 1, pp 1-5

6. Morisky D.E., et al. (2008). Predictive validity of a medication adherence measure in an outpatient setting. The Journal of Clinical Hypertension, 10 (5), pp. 348-354.

7. Pharmacists Association and GreenShield Canada (2014). Impact of Community Pharmacist Interventions in Hypertension Management on Patient Outcomes: A Randomized Controlled Trial Final Project Report. British Journal of Clinical Pharmacology, 78 (6), pp 1238-1247.

8. Sumitra Shrestha (2019). Impact of Pharmacist Counselling on Medication Adherence among Elderly Patients on Antihypertensive Therapy in a Tertiary Care Hospital of Nepal.Europasian Journal of medical Sciences, Vol 1, Jul-Dec 2019.

\section{NGHIÊN CỨU BÀO CHẾ VIÊN NANG CHỨA ESOMEPRAZOL 20MG DẠNG HẠT BAO TAN TRONG RUỘT}

\section{TÓM TẮT}

Muc tiêu: Bào chế viên nang chứa esomeprazol dạng hạt bao tan trong ruột. Đối tượng và phương pháp: Đối tượng nghiên cứu là esomeprazol với polymer Eudragit ${ }^{\circledR}$ L30D-55. Khảo sát lớp bao mang hoạt chất (tỷ lệ cồn: nước, khối lượng PEG 6000, khối lượing talc, hàm lượng chất khô), khảo sát lớp bao cách ly (tỷ lê cồn: nước, khối lượng PEG 6000, hàm lượng chất khô), khảo sát lớp bao tan trong ruột (khối lượng $\mathrm{TiO}_{2}$, độ tăng trọng lớp bao tan trong ruột) đến quá trình tạo hạt nhằm xác định độ hoà tan của hạt trong môi trường acid $\mathrm{HCl} 0,1 \mathrm{~N}$ trong 2 giờ là không quá $10 \%$ và trong môi trường đệm phosphat $\mathrm{pH} 6,8$

*Đai hoc Y Dước Cần Thơ

Chịu trách nhiệm chính: Nguyễn Thị Linh Tuyền

Email: ntltuyen@ctump.edu.vn

Ngày nhận bài: 1/3/2021

Ngày phản biên khoa hoc: 28/3/2021

Ngày duyệt bài: 25/4/2021

\section{Nguyễn Thị Linh Tuyền*, Phan Lê Hoài Ân*}

trong 30 phút không nhỏ hơn $80 \%$. Kết quả: ở lớp bao mang hoạt chất có tỷ lệ cồn: nhước là $3: 1$, khối lượng PEG 6000 là 2,1g, khổi lượng talc là 2,1g, hàm lượng chất khô là $8 \%$. Ơ lớp bao cách ly có tỷ lệ cồn: nước là 3:1, khối lượng PEG 6000 là 1,2g, hàm lượng chất khô là $6 \%$. Ở lớp bao tan trong ruột có khối lượng $\mathrm{TiO}_{2}$ là $1 \mathrm{~g}$, độ tăng trọng của lớp bao tan trong ruột là $30 \%$. Kết luân: đã bào chếviên nang chứa esomeprazol dạng hạt bao tan trong ruột đạt tiêu chuẩn của USP43.

Tư khoá: Esomeprazol, Eudragit ${ }^{\circledR}$ L30D-55, hạt.

\section{SUMMARY}

\section{FORMULATION OF ESOMEPRAZOLE 2OMG DELAYED RELEASE PELLETS CONTAINED CAPSULES}

Objectives: Preparation of capsules containing esomeprazole delayed release pellet. Materials and methods: The materials were esomeprazole with polymer Eudragit ${ }^{\circledR}$ L30D-55. Survey the active coating layer (alcohol: water ratio, PEG 6000 weight, talc 